Elastic Shear Modulus: Fits to Data and

Extrapolation to Large Compressions

and Negative Pressure

Galen K. Straub 


\section{ELASTIC SHEAR MODULUS: FITS TO DATA AND \\ EXTRAPOLATION TO LARGE COMPRESSIONS AND NEGATIVE PRESSURE}

by

Galen K. Straub

ABSTRACT

An analytic form for the elastic shear modulus is presented and its fit to data at different compressions is demonstrated for copper and tungsten. When only the shear modulus at zero pressure and its logarithmic derivative are known, an approximate procedure is used to generate the parameters for an analytic form for the shear modulus. This proceoure is demonstrated and estimates are provided of the shear modulus as a function of compression, for $\mathrm{Al}, \mathrm{Cu}, \mathrm{F}$ e, Mo, W, Ta, and U.

\section{INTRODUCTION}

Experimental data for the elastic shear modulus $G$ as a function of compression are severely limited to values near zero pressure for most metals. Dynamic material models of plastic flow usually require information about the shear modulus over a range of compressions, and sometimes, temperatures. In the present report, we give formulae for the extrapolation of $G$ at fixed temperature over a range of compression including the negative pressure regime.

Data for the shear modulus as a function of compression exist mostly in two 
forms. The most complete set is $G(\rho)$ over a modest compression rillie. For these data, we have fitted a three-parameter analytic function that is accurate over the range of data to a few tenths of a percent. When available, the fitted functions of $G_{f i t}\left(\rho / \rho_{0}\right)$ have high accuracy as extrapolation formulae to large compression and for negative pressures (tension). The second and more common form of the data consists of the shear modulus at zero pressure, $G_{0}$, and a volume derivative. For the cases where only $G_{0}$ and its logarithmic derivative $\mathrm{X}_{0}=(\partial \ln G / \partial \ln \rho)_{0}$ evaluated at pressure $\mathrm{P}=0$ are known, we have used this information to approximate the three parameters in $G_{f i t}$. This second procedure gives results that are accurate to a few percent over a limited compression range around $\rho_{0}$.

In Section II, we present the analytic form for $G$ and the formulae needed to determine its parameters. Section III discusses the approximate determination of the needed parameters using $G_{0}$ and $X_{0}$.

II. THE INTERPOLATION FORMULA FOR $G(\rho)$

In the process of representing the results of eiectronic structure calculations of the energy $E$ as a function of the lattice constant a, a useful analytic form is $(1)$

$E(a)=c\left(1+b\left(a-a_{0}\right)\right) \exp \left(-b\left(a-a_{0}\right)\right)+d$, where $a_{0}, b, c$, and $d$ are fitted to the results. Derivatives of $E(a)$ are given by

$$
\begin{aligned}
& E^{\prime}=-c b^{2}\left(a-a_{0}\right) \exp \left[-b\left(a-a_{0}\right)\right], \\
& E^{\prime}=E^{\prime}\left[1 /\left(a-a_{0}\right)-b\right], \\
& E^{\prime}{ }^{\prime}=E^{\prime}\left[b^{2}-2 b /\left(a-a_{0}\right)\right] .
\end{aligned}
$$

Using these expressions for the energy and its derivatives, the pressure, bulk modulus, and $\partial B / \partial P$ may be determined from

$$
\begin{aligned}
& \mathrm{P}=-\partial \mathrm{E} / \partial \mathrm{V}=-\xi / 3 \mathrm{a}^{2} \mathrm{E}^{\prime}, \\
& \mathrm{B}=-\mathrm{V} \partial \mathrm{P} / \partial \mathrm{V}=-\xi / 9 \mathrm{a} \mathrm{E}^{\prime \prime}+2 \mathrm{P} / 3, \\
& \partial \mathrm{B} / \partial \mathrm{P}=-\mathrm{V} / \mathrm{B} \partial \mathrm{B} / \partial \mathrm{V}=1-2 \mathrm{P} / 9 \mathrm{~B}-\xi / 27 \mathrm{~B} \mathrm{E}^{\prime \prime},
\end{aligned}
$$

where the volume is given by $V=a^{3} / \xi$ and $\xi=2$ for the bcc structure (tungsten) and $\xi=4$ for the fcc structure (copper).

For the shear modulus as a function of the lattice constant a, we used the form 


$$
G=G_{0}+g_{1}\left(a-a_{0}\right) / a^{2} \exp \left[-g_{2}\left(a-a_{0}\right)\right] .
$$

The pressure derivative of the shear modulus is represented as

$$
\mathrm{dG} / \mathrm{dP}=-\mathrm{V} / \mathrm{B} \quad \xi\left(\mathrm{G}-\mathrm{G}_{0}\right) / 3 \mathrm{a}^{2}\left[1 /\left(\mathrm{a}-\mathrm{a}_{0}\right)-2 / \mathrm{a}-\mathrm{g}_{2}\right] \text {. }
$$

The choice of the form for $G(a)$ was motivated by the observation of the similar nature of the electronic structure results for the bulk modulus and the transition metal pseudopotential results of the shear modulus for tungsten at high pressures. The above expressions for $P$ and $B$ suggest that the shear modulus should be well represented by a function of the same form.

The parameters in the interpolation formula for $G\left(a_{0}, G_{0}, g_{1}\right.$, and $\left.g_{2}\right)$ are best determined from a knowledge of $G_{0}$ at $a_{0}, G\left(a_{1}\right)$, and $G\left(a_{2}\right)$. Then, we have

$$
\begin{aligned}
& g_{1}=\left(G\left(a_{1}\right)-G_{0}\right)\left[a_{1}{ }^{2} /\left(a_{1}-a_{0}\right)\right] \exp \left[-g_{2}\left(a_{1}-a_{0}\right)\right] \text { and } \\
& g_{2}=\left(1 /\left(a_{1}-a_{2}\right)\right) \ln \left(\left[G\left(a_{2}\right)-G_{0}\right] /\left[G\left(a_{1}\right)-G_{0}\right]\left(a_{2} / a_{1}\right)^{2}\left(a_{1}-a_{0}\right) /\left(a_{2}-a_{0}\right)\right] .
\end{aligned}
$$

Useful formulae for the derivatives of $G$ are

$$
\begin{aligned}
\partial G / \partial a= & \left(1 / a^{3}\right)\left[-g_{1} g_{2} a\left(a-a_{0}\right)-g_{1}\left(a-2 a_{0}\right)\right] \exp \left[-g_{2}\left(a-a_{0}\right)\right], \\
\partial^{2} G / \partial a^{2} & =\left(1 / a^{4}\right)\left[g_{1} g_{2}{ }^{2} a^{2}\left(a-a_{0}\right)+2 g_{1} g_{2} a\left(a-2 a_{0}\right)+2 g_{1}\left(a-3 a_{0}\right)\right] \exp \left[-g_{2}\left(a-a_{0}\right)\right], \\
\partial^{3} G / \partial a^{3} & =\left(1 / a^{5}\right)\left[-g_{1} g_{2}{ }^{3} a^{3}\left(a-a_{0}\right)-3 g_{1} g_{2} a^{2}\left(a-2 a_{0}\right)-6 g_{1} g_{2} a\left(a-3 a_{0}\right)-6 g_{1}\left(a-4 a_{0}\right)\right] \\
\bullet & \exp \left[-g_{2}\left(a-a_{0}\right)\right] .
\end{aligned}
$$

As a increases, G initially decreases with the minimum at

$$
\mathrm{a}=\left(\left(\mathrm{a}_{0} \mathrm{~g}_{2}-1\right)+\left[\left(\mathrm{a}_{0} \mathrm{~g}_{2}\right)^{2}+6 \mathrm{a}_{0} \mathrm{~g}_{2}+1\right]^{1 / 2}\right) / 2 \mathrm{~g}_{2} .
$$

In copper, this corresponds to $\rho / \rho_{0}=0.53$. This is well beyond a meaningful extrapolation of the data and in the regime of possible metal-insulator transitions; the form for $G$ presented here needs further study in this extreme low density region. A reasonable procedure would be to require $G$ to quickly approach zero for $\rho / \rho_{0}<0.8$.

The results for the fits for tungsten and copper are shown in Fig. 1 where the solid circles and diamonds are the fitted points. Values of $g_{1}$ and $g_{2}$ are given in Table 1. The accuracy over the entire range for both materials is on the order of a few tenths of a percent. Eq.(1) also gives an excellent representation of the data in the negative pressure regime without additional approximations. A more extensive analysis of the elastic propesties of copper is given in Refs. 1 and 2 .

For reference, the lattice constant and the density are related by volume/unit mass $=v=1 / \rho=a^{3} /\left(\hat{\xi}\right.$ Atw $\left./ \mathrm{N}_{0}\right)$,

where Atw is the atomic weight per mole, $N_{0}=6.02252 \times 10^{23} / \mathrm{mole}$ is Avogadro's number, and $\xi=$ the number of atoms in the unit cell. For $\rho$ in grams per cubic 
centimeter and Atw in grams,

$$
\begin{aligned}
a^{3} & =\xi \text { Atw } /\left[\rho\left(6.02253 \times 10^{23}\right)\left(0.529167 \times 10^{-8} \mathrm{~cm} / \alpha\right)^{3}\right] \\
& =\alpha^{3} 11.206 \xi \text { Atw } / \rho,
\end{aligned}
$$

giving $a$ in units of $\alpha=$ Bhor radius.

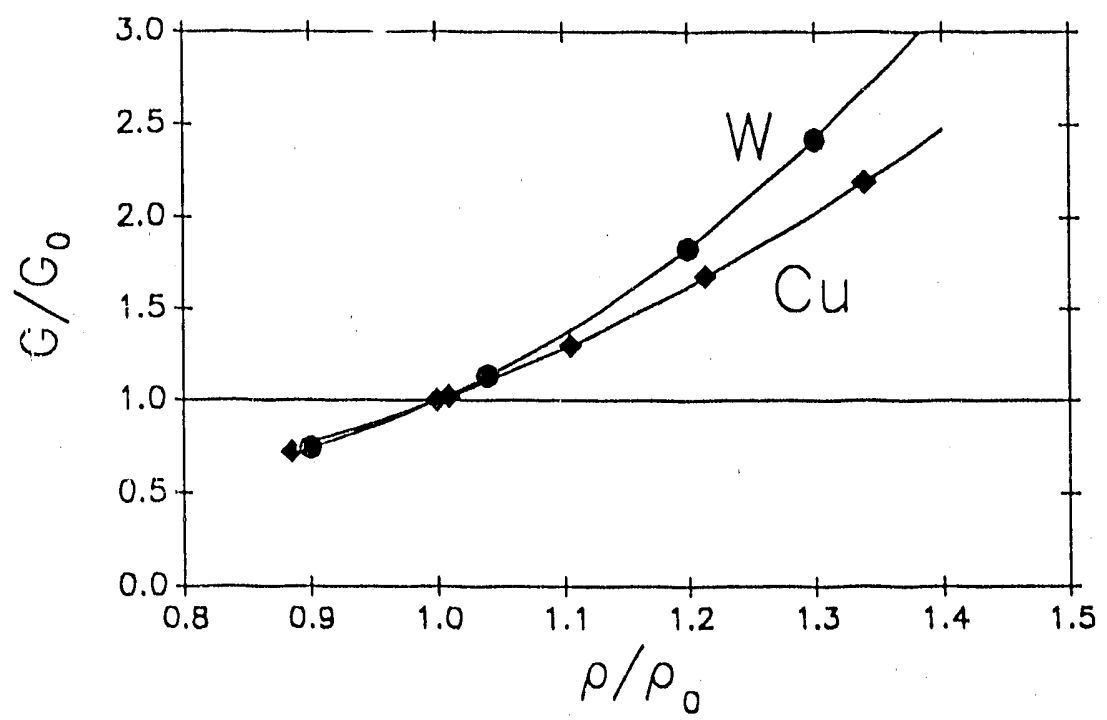

Figure 1

The shear modulus $G$ vs compression for copper and tungsten. The circles and diamonds represent selected values of the shear modulus, ( 1 ) and the solid line is the result of Eq. ( 1 ).

TABLE 1. Fitted parameters for $G=G_{0}+g_{1}\left(a-a_{0}\right) / a^{2} \exp \left[-g_{2}\left(a-a_{0}\right)\right]$ where $g_{1}$ is in units of Mbar $-\alpha$ and $g_{2}$ is in units of $1 / \alpha\left(\alpha=\right.$ Bohr radius $=0.529167^{2} \mathrm{x}$ $\left.10^{-8} \mathrm{~cm}\right)$.

$$
\begin{array}{cccc}
\rho_{0} & \mathrm{G}_{0} & \mathrm{~g}_{1} & \mathrm{~g}_{2} \\
\left(\mathrm{~g} / \mathrm{cm}^{3}\right) & (\mathrm{Mbar}) & (\mathrm{Mbar}-\alpha) & (1 / \alpha)
\end{array}
$$

$\begin{array}{lllll}\mathrm{Cu} & 9.020 & 0.518 & -26.7006 & 0.529256 \\ \mathrm{~W} & 19.3 & 1.629 & -89.4752 & 0.877346\end{array}$


III. EXTRAPOLATIONS OF THE SHEAR MODULUS FROM $G_{O}$ AND ITS LOGARITHMIC DERIVATIVE

For many metals, the only information available is the shear modulus at zero pressure, $G_{0}$, and the logarithmic derivative $X_{0}=(\partial \ln G / \partial \ln \rho)_{0}=$ $\left(B_{0} / G_{0}\right)(\partial G / \partial P)_{0}$. We can use $X_{0}$ to make an approximation to the parameters $g_{1}$ and $\mathrm{g}_{2}$ by assuming that near $\mathrm{a}_{0}, \mathrm{G} \propto \rho^{\mathrm{X}_{0}}$. Since $\rho \propto \mathrm{a}^{3}$, then $\mathrm{d}(\ln \rho)=-3 \mathrm{~d}(\ln$ a). Equating the first and second lerivatives of $G$ from $\mathrm{Eq} .(1)$ and $G \propto a^{-3 X_{0}}$, we obtain

$$
\begin{aligned}
& (\partial \mathrm{G} / \partial \mathrm{a})_{\mathrm{a} O}=-3 \mathrm{X}_{0} \mathrm{G}_{0} / \mathrm{a}_{0}=\mathrm{g}_{1} / \mathrm{a}_{0}{ }^{2} \text { and } \\
& \left(\partial^{2} \mathrm{G} / \partial \mathrm{a}^{2}\right)_{\mathrm{a} O}=3 \mathrm{X}_{0} \mathrm{G}_{0} / \mathrm{a}_{0}{ }^{2}\left(1+3 \mathrm{X}_{0}\right)=-2 \mathrm{~g}_{1} / \mathrm{a}_{0}\left(\mathrm{~g}_{2}+2 / \mathrm{a}_{0}\right) .
\end{aligned}
$$

Solving for $g_{1}$ and $g_{2}$ gives

$$
\begin{aligned}
& g_{1}=-3 a_{0} G_{0} x_{0} \text { and } \\
& g_{2}=3\left(x_{0}-1\right) / 2 a_{0} .
\end{aligned}
$$

Table 2 gives the approximate values of $g_{1}$ and $g_{2}$ using the logarithmic derivative and data supplied by D. C. Wallace and D. L. Preston (X-4, Los Alamos National Laboratory). Figure 2 plots $G / G_{0}$ for $A 1, W, C u, F e, M o$, and Ta.

For copper and tungsten, we can compare fits of $G(\rho)$ with the approximation of the first and second derivatives from $X_{0}$. This is shown in Fig. 3 . At a compression of 1.04 , the differences between the two results are 4.68 and 6.18 for $\mathrm{Cu}$ and $\mathrm{W}$, respectively. The difference is approximately 1 inear with compression for $0.9<\rho / \rho_{0}<1.1$.

The analytic form for the shear modulus (Eq. (1)) has been studied for its validity to a compression of 10 . The behavior of the materials listed in Table 2 is still metallic; the electronic structure is dominated by a nearly-free electron gas and an array of ions screened by the electrons. (3) Even the lightest metals in the periodic chart retain at least the 1 s core staces and the effective ion-ion interaction requires the inclusion of electron screening in a nonuniform electron gas. (4) The pressure at a compression of 10 is of the order of a few thousand megabars. The limit as $\rho$ goes to infinity is a much more difficult problem requiring an accurate quantum mechanical, relativistic treatment of all electron states and remains an open question. (5) The use of Eq. (1) for $G$ is appropriate for most high density applications.

At negative pressures, the analytic form for $G$ should also be accurate to the point where fracture occurs. Eq. (1) accurately reproduces the available values of $G$ for tungsten and copper to $\rho / \rho_{0}$ equal to 0.91 and 0.85 , respectively. The behavior of the other metals should be qualitatively similar. 


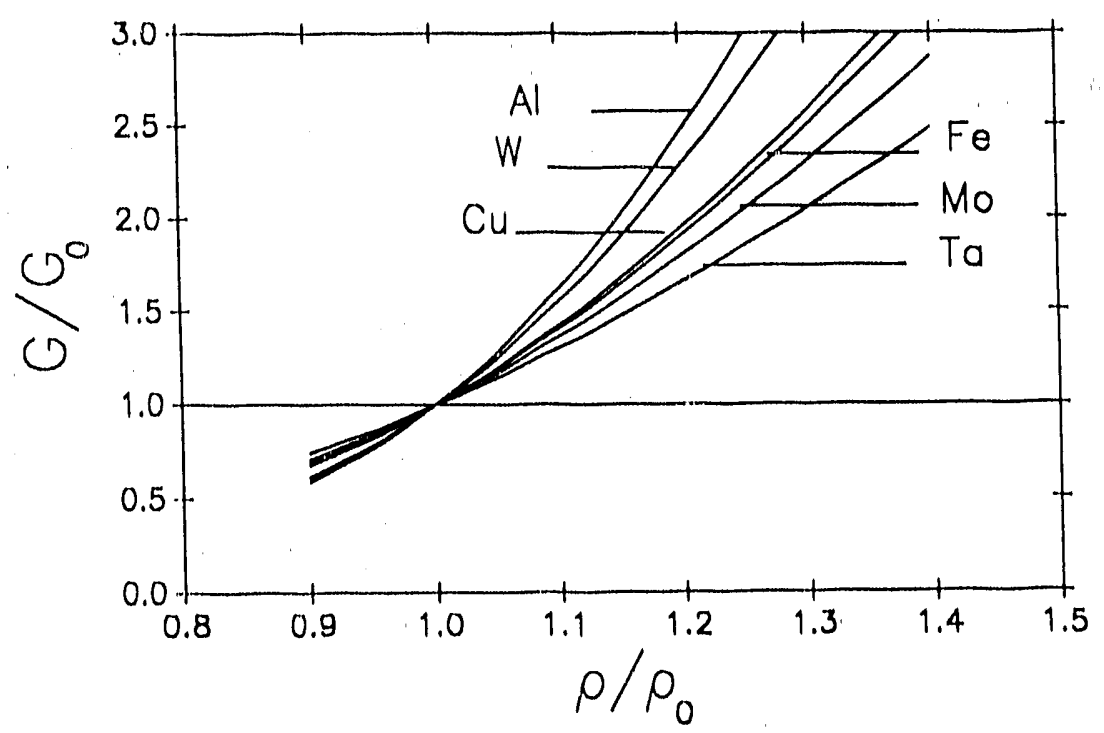

Figure 2.

The shear modulus $\mathrm{G}$ as a function of compression using Eq. (1) with parameters $\mathrm{g}_{1}$ and $g_{2}$ determined from the logarithic derivative $x_{0}$.

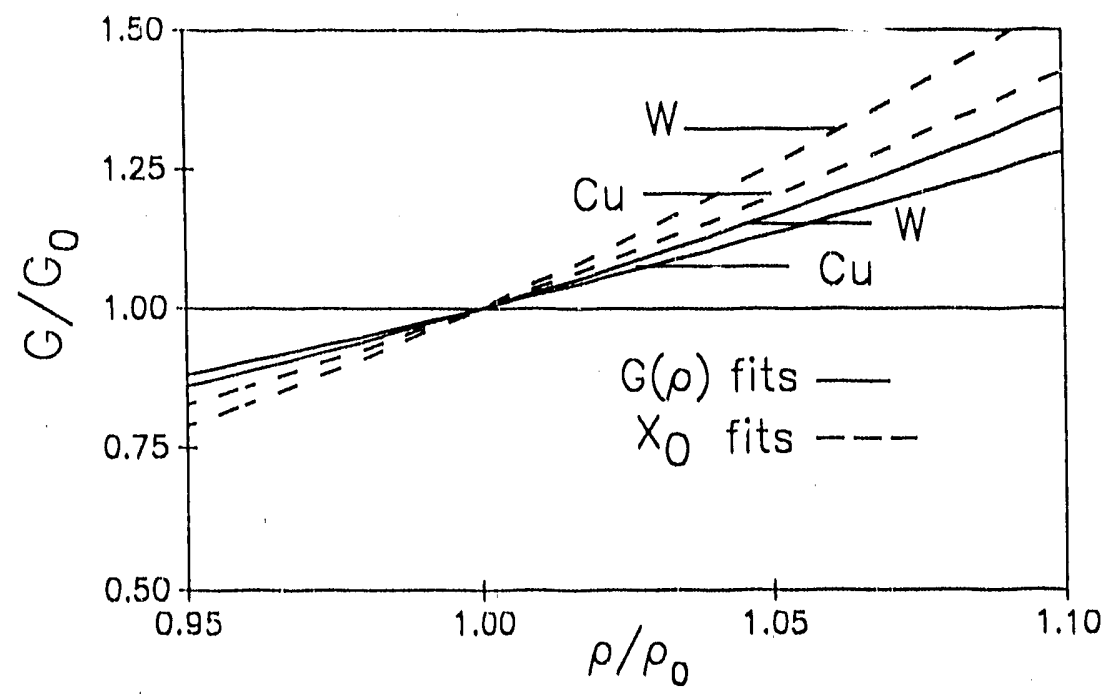

Figure 3.

Comparisons between the shear modulus $(\mathrm{f}$ fitted to data at different compressions and fits determined using the logarithmic derivative $\mathrm{x}_{0}$ 
TABLE 2. Experimental data and $g_{1}$ and $g_{2}$ values determined from the logarithmic derivative $\left(\alpha=\right.$ Bhor radius $\left.=0.529167 \times 10^{-8} \mathrm{~cm}\right)$.

\begin{tabular}{lcccccc} 
Metal & $\xi$ & $\begin{array}{c}\rho_{0} \\
\left(\mathrm{~g} / \mathrm{cm}^{3}\right)\end{array}$ & $\begin{array}{c}\mathrm{G}_{0} \\
(\mathrm{Mbar})\end{array}$ & $\mathrm{x}_{0}$ & $\begin{array}{c}\mathrm{g}_{1} \\
(\mathrm{Mbar}-\alpha)\end{array}$ & $\begin{array}{c}\mathrm{g}_{2} \\
(1 / \alpha)\end{array}$ \\
\hline $\mathrm{Al}$ & 4 & 2.734 & 0.293 & 5.1 & -34.1574 & 0.80714 \\
$\mathrm{Cu}$ & 4 & 9.02 & 0.518 & 3.73 & -39.4709 & 0.601363 \\
$\mathrm{Fe}$ & 2 & 7.92 & 0.872 & 3.6 & -50.9165 & 0.72135 \\
$\mathrm{Mo}$ & 2 & 10.27 & 1.303 & 3.27 & -75.9014 & 0.57343 \\
$\mathrm{~W}$ & 2 & 19.3 & 1.634 & 4.65 & -136.235 & 0.916056 \\
$\mathrm{Ta}$ & 2 & 16.8 & 0.722 & 2.8 & -37.7623 & 0.433633 \\
$\mathrm{U}$ & 4 & 19.2 & 0.938 & 3.6 & -83.2863 & 0.47437 \\
\hline
\end{tabular}


TABLE 3. Values of $\mathrm{G} / \mathrm{G}_{0}$ as a function of compression. All values in this table were determined from approximations using $\mathrm{X}_{0}$.

\begin{tabular}{llllllll}
\hline$\rho / \rho 0$ & $\mathrm{~A} 1$ & $\mathrm{Cu}$ & $\mathrm{Fe}$ & $\mathrm{Mo}$ & $\mathrm{W}$ & $\mathrm{Ta}$ & $\mathrm{U}$ \\
\hline 0.900 & 0.591 & 0.678 & 0.687 & 0.711 & 0.618 & 0.746 & 0.687 \\
0.920 & 0.657 & 0.734 & 0.742 & 0.762 & 0.681 & 0.792 & 0.742 \\
0.940 & 0.731 & 0.795 & 0.801 & 0.817 & 0.751 & 0.841 & 0.801 \\
0.960 & 0.812 & 0.859 & 0.864 & 0.875 & 0.827 & 0.892 & 0.864 \\
0.980 & 0.902 & 0.927 & 0.930 & 0.936 & 0.910 & 0.945 & 0.930 \\
1.000 & 1.000 & 1.000 & 1.000 & 1.000 & 1.000 & 1.000 & 1.000 \\
1.020 & 1.106 & 1.077 & 1.074 & 1.067 & 1.096 & 1.057 & 1.074 \\
1.040 & 1.221 & 1.157 & 1.151 & 1.137 & 1.200 & 1.116 & 1.151 \\
1.060 & 1.344 & 1.242 & 1.233 & 1.209 & 1.310 & 1.1 .77 & 1.233 \\
1.080 & 1.477 & 1.331 & 1.318 & 1.285 & 1.427 & 1.240 & 1.318 \\
1.100 & 1.618 & 1.424 & 1.407 & 1.364 & 1.552 & 1.305 & 1.407 \\
1.120 & 1.768 & 1.521 & 1.499 & 1.445 & 1.683 & 1.371 & 1.499 \\
1.140 & 1.928 & 1.622 & 1.595 & 1.529 & 1.822 & 1.440 & 1.595 \\
1.160 & 2.097 & 1.727 & 1.695 & 1.616 & 1.968 & 1.510 & 1.695 \\
1.180 & 2.276 & 1.836 & 1.798 & 1.706 & 2.122 & 1.582 & 1.798 \\
1.200 & 2.464 & 1.949 & 1.905 & 1.798 & 2.283 & 1.656 & 1.705 \\
\hline & & & & & & & \\
\hline
\end{tabular}




\section{REFERENCES}

1. G. K. Straub and J. M. Wills, "Elastic Moduli of Copper: Electronic Structure Contributions Under Compression," LA-11522-MS (November 1989).

2. G. K. Straub, J. M. Wills, and D. C. Wallace, "Elastic Moduli of Copper Under Compression," in Shock Waves in Condensed Matter .- 1987, edited by S.C. Schmidt and N. C. Holmes (Elesevier Science Publishers, B. V., 1988).

3. G. K. Straub and W. A. Harrison, "Analytic Methods for the Calculation of the Electronic Structure of Solids," Phys. Rev. B $\underline{31}, 7668$ (1984).

4. G. K. Straub, "The Overlap of Electron Core States for Very Higl Compressions," in Shock Waves in Condensed Matter -. 1985, edited by Y. M. Gupta (P1enum Press, 1986).

5. K. Moulopoulos and N. W. Ashcroft, "Scaling Relations for Two-component Charged Systems: Application to Metallic Hydrogen," Phys. Rev. B 41, 6500 (1990). 
-END-

DATE FILMED

$01 / 22 / 91$ 
I. 